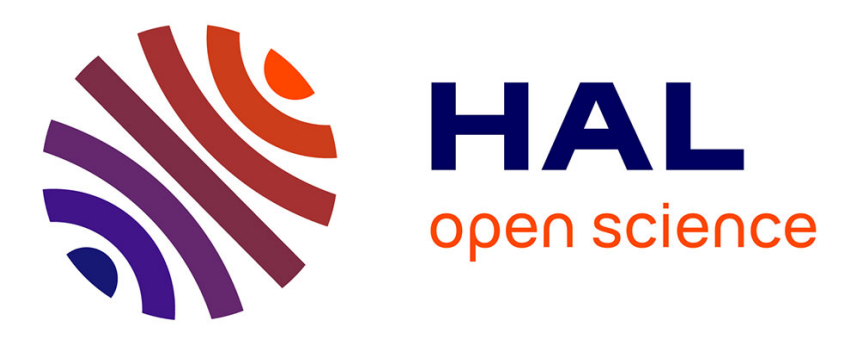

\title{
Quantifying the flexibility of a manufacturing system by applying the transfer function
}

Kosmas Alexopoulos, Nikolaos Papakostas, Dimitris Mourtzis, Pantelis Gogos, George Chryssolouris

\section{- To cite this version:}

Kosmas Alexopoulos, Nikolaos Papakostas, Dimitris Mourtzis, Pantelis Gogos, George Chryssolouris. Quantifying the flexibility of a manufacturing system by applying the transfer function. International Journal of Computer Integrated Manufacturing, 2007, 20 (06), pp.538-547. 10.1080/09511920600930046 . hal-00513383

\section{HAL Id: hal-00513383 \\ https://hal.science/hal-00513383}

Submitted on 1 Sep 2010

HAL is a multi-disciplinary open access archive for the deposit and dissemination of scientific research documents, whether they are published or not. The documents may come from teaching and research institutions in France or abroad, or from public or private research centers.
L'archive ouverte pluridisciplinaire HAL, est destinée au dépôt et à la diffusion de documents scientifiques de niveau recherche, publiés ou non, émanant des établissements d'enseignement et de recherche français ou étrangers, des laboratoires publics ou privés. 


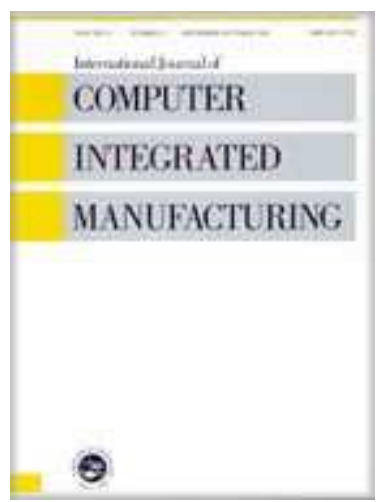

\section{Quantifying the flexibility of a manufacturing system by applying the transfer function}

\begin{tabular}{|r|l|}
\hline Journal: & International Journal of Computer Integrated Manufacturing \\
\hline Manuscript ID: & TCIM-2005-IJCIM-0062.R2 \\
\hline Manuscript Type: & Original Manuscript \\
\hline Date Submitted by the & 22-Jul-2006 \\
\hline Complete List of Authors: & $\begin{array}{l}\text { Alexopoulos, Kosmas; University of Patras, Laboratory for } \\
\text { Manufacturing Systems and Automation } \\
\text { Papakostas, Nikolaos; University of Patras, Laboratory for } \\
\text { Manufacturing Systems and Automation } \\
\text { Mourtzis, Dimitris; University of Patras, Laboratory for } \\
\text { Manufacturing Systems and Automation } \\
\text { Gogos, Pantelis; University of Patras, Laboratory for Manufacturing } \\
\text { Systems and Automation } \\
\text { Chryssolouris, George; University of Patras, Laboratory for } \\
\text { Manufacturing Systems and Automation }\end{array}$ \\
\hline Keywords: & \begin{tabular}{l} 
MANUFACTURING SYSTEMS, FLEXIBILITY MEASURES, FLEXIBILITY \\
\hline Keywords (user):
\end{tabular} \\
\hline
\end{tabular}

\section{S ScholarONE" \\ Manuscript Central}




\title{
Quantifying the flexibility of a manufacturing system by applying the transfer function
}

\author{
K. ALEXOPOULOS, N. PAPAKOSTAS, D. MOURTZIS, P. GOGOS, \\ G. CHRYSSOLOURIS* \\ Laboratory for Manufacturing Systems and Automation, \\ Director Prof. George Chryssolouris \\ Department of Mechanical Engineering and Aeronautics \\ University of Patras, 26500 Rio, Patras, Greece. \\ E mail: gchrys@hol.gr,www.lms.mech.upatras.gr \\ Tel.: +302610 997262, Fax: +302610997744
}

This paper discusses a method of estimating the flexibility of a manufacturing system. The approach proposed, is motivated by the dynamic behaviour analogy between a mechanical and a manufacturing system. The main hypothesis is that the flexibility of a manufacturing system can be calculated, in the same manner, as the damping factor of a mechanical system. In order for the validity of the method proposed, to be tested and discussed, a set of experiments has been designed and executed, initially, for a simple production system. An industrial production system has also been studied having used the same approach. The results prove that this method can be used for the estimation of a manufacturing system's flexibility.

\footnotetext{
*Corresponding author - email: gchrys@ hol.gr
} 


\section{Introduction}

Shorter product lifecycles and the increased number of new models and variants have forced companies to produce products that would meet the demands of a diversified customer base, in a short development cycle, yielding low cost, high quality and sufficient quantity. This makes manufacturing flexibility an increasingly important attribute of modern manufacturing systems (Chryssolouris 2005). The capacity of profitably absorbing fluctuations in demand, so as to develop and bring in new products quicker by using existing facilities, is considered an important attribute of modern manufacturing firms (Beach et al. 2000). However, flexibility cannot be properly considered in the decision making process, if it is not properly defined in a quantified fashion.

Several methods of measuring manufacturing system flexibility have been proposed. Kumar (1987) used the entropy concept of thermodynamics that provides similarities to flexibility measures; the entropy concept has been recently revised by Chang et al. (2001). Alternatively Brill and Mandelbaum (1989) suggested that flexibility can be estimated relatively to a reference task set and Gupta (1993) employed financial analysis. More recently, Boyer and Leong (1996) proposed the changeover cost as measure of machine flexibility and they examine how machine flexibility is related to process flexibility. Tsourveloudis and Phillis (1998) and Beskese et al. (2004) have employed fuzzy logic rules that include engineers and managers' expertise for measuring machine flexibility. Khouja (1998) has studied volume flexibility of a system by examining the cost of the shape per piece curve of the system for different production volumes. Shewchuk (1999) developed a set of generic application measures for the evaluation of different flexibility types. Routing flexibility is represented by 
using direct acyclic graphs that enumerate all possible manufacturing operation sequences that can be applied to a certain part (Borenstein (2000)). Garavelli (2001) investigates the benefits of a different degree of routing flexibility by measuring both the lead-time and the work-in-process performance for different system configurations. In Ramasesh et al. (2001) the value of flexibility has been explored in financial terms and they suggest an aggregate metric of flexibility, in terms of the expected net present value of all relevant cash flows, associated with a system's response to random, unanticipated changes. Bengtsson and Olhager (2002) use real options to assess product-mix flexibility concerning multiple products, capacity constraints, and set-up costs, with respect to the level of automation and the multi-functionality of resources. Koren et al. (2003) proposed a measure for evaluating the convertibility of manufacturing systems which considers configuration, machine and material handling level. Kurtoglu (2004) has studied the flexibility of two assembly lines, by calculating the manufacturing costs of changing a system in order to produce new products. Hop (2004) measures the product mix flexibility response in terms of both capability, which is represented with respect to the number of operations that a machine can perform, and capacity, which is modelled in terms of the efficiency of different machines. Wiendahl and Heger (2004) proposed a method of justifying changeability in economical terms, using the scenario planning technique. Wahab (2005) has studied measures for machine and product mix flexibility response under dynamic manufacturing environments, based on machine-operation efficiency and tooling requirements. Recent reviews on different approaches for assessing flexibility can be found in Barad and Nof (1997), De Toni and Tonchia (1998) and Beach et al. (2000). 
Chryssolouris and Lee (1992) and Chryssolouris (1996) suggested that the 'flexibility of a manufacturing system is determined by its sensitivity to change' and it is evaluated by calculating the expected cost of accommodating possible changes in the operating environment. The smaller the expected change cost is, the less sensitive the system is to changes in its operating environment and thus, the system is considered as more flexible. Bateman et al. (1999) have extended the 'sensitivity to change' approach while Alexopoulos et al. (2005) have applied this approach to a real case study of the commercial refrigerators production industry. Although, this method may provide practical flexibility indication for mid and long-term decisions, it cannot be used for short-term decisions as it requires estimates for future market requirements that are sometimes rather difficult to acquire and their accuracy may be questionable. Moreover, Chryssolouris et al. (1998) have proposed a measure of flexibility, based on the calculation of the transfer function of the manufacturing system. The 'transfer function' describes the way a manufacturing system responds to changes in the input i.e., to various orders of different products and to different volumes, in the same manner as the transfer function characterizes a simple mechanical system, which responds under the excitation of a force vibrating over time. In a mechanical system, the damping factor reflects the capability of a system to respond to changes in the excitation and it can be calculated via its transfer function under certain circumstances (Seybert 1981). In the same manner, the flexibility of a manufacturing system reflects the ability of a system to respond to a dynamic input (see figure 1). Motivated by this idea, a measure of flexibility can be calculated.

[Insert figure 1 about here] 
Although most of the research efforts presented above may be adequate for different industrial situations, their application is usually restricted to specific industrial cases and thus, they are not generally applicable. Additionally, they usually require rather complex data, difficult to find in real industrial environment, and it becomes a strong barrier in their everyday application in industrial practice. On the other hand, the proposed measure of flexibility, differentiates with most of the existing flexibility measures, in the following ways:

- It utilizes rather simple data that are usually available in the modern ERP systems.

Such data describe material or information flow that may be either input or output from the manufacturing system.

- It is generic in the sense that it is not bound to some specific flexibility type. Consequently, it may be applicable to different industrial situations by eliminating the need for identifying a suitable flexibility measure in a given situation.

\section{A flexibility index using the mechanical analogy concept - FLEXIMAC}

In the current work, the input/excitation is the Processing Time (PT) of parts produced and the output/response is the Flow Time (FT) of the parts. PT is the time required for a part to be produced by the system, at a given time, while FT is the flow time of the same part at the given time. The reasoning for selecting PT as input to the manufacturing system and FT as output of the system is as follows: PT can be viewed as a kind of 'excitation' to the system since it represents the demand to the system. PT is calculated assuming 'pure' processing times without any set up times and delays resulting from 
waiting for the resources of the system to become available. On the other hand FT includes the required set up and waiting times and as such can be viewed as the response of the system to the demand. In ideal situations, FT may be identical to PT, in case the system does not require any set ups and the resources are always available to process the parts. Thus the differences between PT and FT constitute the ability of the system to respond to a given demand. The better the response, the smaller the differences.

In order for the value of FLEXIMAC to be calculated, the following steps take place:

1. At equally spaced time intervals $(\Delta t)$, record the parts exiting the system (after their processing has finished)

2. As input to the system, at each recording period, is considered the sum of PTs of the recorded parts and as output the sum of FTs of the recorded parts.

3. Calculate the transfer function of the production system, in the frequency domain, from the recorded input-output data for all recording periods.

4. Calculate the value of FLEXIMAC from the transfer function, in the same manner as the damping factor of a mechanical system is calculated.

These steps are described below in more detail.

At equally spaced time intervals $\Delta t$ the parts that have been produced by the system during $\Delta t$ are recorded. Then the Cumulative Processing Time (CPT) and the Cumulative Flow Time (CFT) are calculated for each time interval. CPT and CFT are the sum for PT and FT, respectively, for all parts produced within a given $\Delta t$. Consequently, the values of CPT and CFT are recorded at constant time intervals $\Delta t$ and totally $N$ CPT and CFT values are recorded:

$\mathrm{CPT}_{\mathrm{k}}, \mathrm{CFT}_{\mathrm{k}}, \mathrm{k}=0,1,2 \ldots \mathrm{N}-1$ 
Moreover:

$\mathrm{CPT}_{\mathrm{k}}=\sum_{i=1}^{M_{k}} P T_{i}$ and $\mathrm{CFT}_{\mathrm{k}}=\sum_{i=1}^{M_{k}} F T_{i}$

where:

$\mathrm{CPT}_{\mathrm{k}}$ is the Cumulative Processing Time at the $\mathrm{k}^{\text {th }}$ time interval

$\mathrm{CFT}_{\mathrm{k}}$ is the Cumulative Flow Time at the $\mathrm{k}^{\text {th }}$ time interval

$M_{\mathrm{k}}$ is the number of parts produced during the $\mathrm{k}^{\text {th }}$ time interval

$\mathrm{PT}_{\mathrm{i}} / \mathrm{FT}_{\mathrm{i}}$ are the $\mathrm{PT} / \mathrm{FT}$ for the $\mathrm{i}^{\text {th }}$ part produced in the $\mathrm{k}^{\text {th }}$ time interval

The transfer function $\mathrm{H}_{\mathrm{n}}(\omega)$ of the system is calculated for a given pair of RPT/CFT data sets:

$$
H_{n}(\omega)=\frac{C F T_{n}(\omega)}{C P T_{n}(\omega)}
$$

where $C F T_{n}$ and $C P T_{n}$ are the Discrete Fourier Transforms of $C F T_{\mathrm{k}}$ and $C P T_{\mathrm{k}}$, respectively, or

$$
\operatorname{CFT}_{n}(\omega)=\sum_{k=0}^{N-1} C F T_{k} e^{\frac{-2 \pi i}{N} n k}, C P T_{n}(\omega)=\sum_{k=0}^{N-1} C P T_{k} e^{\frac{-2 \pi i}{N} n k}
$$

The frequency $\omega$ is given by the relation, $\omega=\omega_{n}=2 \pi n / N, n=0,1,2, \ldots N-1$.

FLEXIMAC is calculated by finding the system eigenvalues $\Omega_{\mathrm{i}}$ and calculating the amplitude $Q_{\mathrm{i}}$ on those $\Omega_{\mathrm{i}}$ frequencies. It must be noted that at $\Omega_{\mathrm{i}}$ the phase angle changes sign and thus a criterion for the identification of $\Omega_{\mathrm{i}}$ can be established (see Chryssolouris et al. (1998)). FLEXIMAC is then calculated as an average value of the ten largest $Q_{\mathrm{i}}$.

FLEXIMAC $=\frac{1}{10} \sum_{i=1}^{10}\left(\frac{1}{2 Q_{i}}\right)$ 
Based on the definition of FLEXIMAC given above, FLEXIMAC is a dimensionless factor, in the same manner as the well-defined in literature, quality measurement, named as process capability index $C_{\mathrm{p}}$ (see Chryssolouris (2005)). FLEXIMAC can be valuable for comparing different production systems when they are exposed to the similar excitation from the external environment. Thus, the value of FLEXIMAC for one system can only be rated relatively to the FLEXIMAC value of other systems, if a similar excitation is imposed upon all systems. The higher the value of FLEXIMAC is in a manufacturing system, the less sensitive the system may be considered to changes in the input and thus, more flexible. Moreover, if the FLEXIMAC value of a system is equal to zero this means that even small excitation input to the system generates very large output and consequently, the system is very sensitive to changes at its input. Additionally, FLEXIMAC can also be considered as a measure of responsiveness, since the smaller the difference between CFT and CPT values the more responsive the system may be considered; the more responsive a system the more flexible. In the following paragraphs, FLEXIMAC is calculated for a simple and a real production system.

\section{Simple production system}

A simple production system having 10 machines working in parallel and producing 10 different products has been designed and implemented, in a simulated mode for testing the validity of the proposed FLEXIMAC factor. Depending on the configuration of the system, one machine can process one or more different parts. Stated otherwise, there is more than one route for the parts. Routing flexibility is the ability to produce a part by alternative routes (Browne et. al. 1984, Stecke and Browne 1985). Routing flexibility 
provides the ability to handle breakdowns and to continue producing a given set of part types. Different configurations have a different degree of routing flexibility and consequently, in this context, FLEXIMAC is used in order to assess it. Moreover, it is assumed that the machines of the system are always available and never kept idle, provided there is a workload waiting. Totally, three different configurations (see figures 2a-c), with increasing flexibility, have been simulated:

A. Low flexibility configuration: Each machine can only process one product.

B. Medium flexibility configuration: Each machine can process three different products.

C. High flexibility configuration: Each machine can process all 10 different products.

[Insert figures 2a-c about here]

A set of experiments has been designed for the study of the effect on the value of the FLEXIMAC factor for obviously different levels of the existing flexibility. It was expected that as the configuration of the system changed towards increasing flexibility, the value of the FLEXIMAC factor would also be increased.

The input to the system is a set of orders for the 10 products. The inter-arrival times for each of the ten products is given by an exponential distribution with a mean value of $\lambda=40$ time units. Each product has a process time, independent of the machine that is going to process it. The process times of all ten products were provided by a normal distribution with a mean $\mu=30$ time units and standard deviation $\sigma=6$. 
For each different configuration, 10 repetitions were made, thus, in total 30 different simulation experiments were performed. In the experiments, 'First-In FirstOut' the shop-floor control policy was used for selecting the orders to be processed by the machines.

The flexibility estimator FLEXIMAC was calculated as follows: The values of the CPT and the CFT were stored for each time interval $\Delta t=30$ time units (see figure 3). The CPT and CFT were expressed in minutes but other time units could be applicable as well. The transfer function of the system was calculated according to (1) and the FLEXIMAC value was calculated from the eigenvalues of the transfer function. In each row of table 1 the values of FLEXIMAC for 10 experiments are presented.

[Insert figure 3 about here]

The value of FLEXIMAC increases when the system becomes more flexible (see table 1). That is, the higher the FLEXIMAC value of a system, the more flexible the system is, whilst the lower the value of the system under consideration, the less flexible it is. The results present the validity of FLEXIMAC.

[Insert table 1 about here]

In figure 4, the value of FLEXIMAX for different levels of flexibility (including the levels in figures $2 \mathrm{a}-\mathrm{c}$ ) is presented. The level of flexibility in each configuration is determined by the average number of possible assignments of products to machines. The results in figure 4, show how FLEXIMAC reflects the changes in the level of 
flexibility. Additionally, in figure 4, the Average Work-In-Process (AWIP) is plotted along with FLEXIMAC. AWIP indicates the ability of the system to keep, in low levels, the number of parts in the system by balancing the load of the machines, due to the routing flexibility. .

[Insert figure 4 about here]

The results in figure 4 indicate that FLEXIMAC is sensitive to the changes in the level of routing flexibility from level 1 up to level 4, whereas there are no significant changes in its values from level 4 to 10 . In the latter case, given the specific workload, the system manages to respond quickly, by balancing the load of the machines, to the input and practically all the parts are processed immediately by the time they arrive at the system. This is also evident from the values of AWIP. Consequently, one could suggest that for the given workload, there is no need to invest in additional flexibility than level 4 does since the performance of the system remains practically unchanged.

\section{Simple production system with breakdowns}

In order for the value of FLEXIMAC to be studied with regards to changes to the availability of the machines of the system, another set of simulation experiments has been executed. The configuration of production systems described in paragraph 3, has been modified so as for the flexibility of the alternative configurations to be measured in case of breakdowns. The experiments have been designed in such a way so as for the observed changes in the behaviour of the system to be depending only on the level of the system's flexibility. 
In all configurations $\mathrm{A}, \mathrm{B}$ and $\mathrm{C}$, each product will follow the route defined in configuration A (i.e. Product 1 to Machine 1, Product 2 to Machine 2 etc.) unless the corresponding machine has been broken-down. In that case, an alternative route as allowed by each of the configurations $\mathrm{B}$ to $\mathrm{C}$ may be followed by the product. Thus, if no breakdowns occur, all four configurations will have the same behaviour and the same FLEXIMAC value if the same input is applied. Should there be breakdowns though, then the behaviour of the configuration will also change. The change in the FLEXIMAC value occurs only due to the different flexibility available at each configuration and not to other reasons (e.g. different production rate).

The same input profile and number of experiments, as in paragraph 3, have been executed and the results are shown in table 2. The results in table 2 show that the value of FLEXIMAC increases as the flexibility of the system increases.

[Insert table 2 about here]

\section{Industrial production system}

Apart from the simple case described above, a real industrial case of a manufacturing system, producing commercial refrigerators and freezers, has been studied. The schematic diagram of the production system is shown in figure 5. The raw materials go through cutting, punching and bending departments, where the first formation of parts takes place. Then the parts end up in a buffer in order for the painting, the insulation and the assembly of internal and external cabins to follow, whilst the parts with surfaces that need printing, go through the printing department. Some parts have to pass through 
the cooling, the electrical, and the doors/shelves departments. At the end, all the parts pass through the final assembly department, where the final product is assembled in an assembly line.

[Insert figure 5 about here]

This study focuses on the punching department of this production system. This case has also been studied in Alexopoulos et al. (2005) but from a mid to long-term flexibility evaluation point of view, mainly for investment evaluation, using the Penalty Of Change method. In this department, the flat metal blanks are processed (punched) before they continue to next step of the bending/folding (transformation to 3Dimensional objects). The punches are producing not only holes (which are used at a later stage for the functionality of the final products, such screwing or riveting of various components) but also the necessary notches of the unfolded - development of the metal sheet. All the parts of the cabinets are processed in the punching department as determined by the products' nature/design, which is the cornerstone of the rest process, as a high volume / efficiency is necessary to catch up with the rest factory. More specifically, three alternatives, having a different degree of routing flexibility due to the technology used, are analysed using the FLEXIMAC concept.

- Press - Press: The department consists of two presses. Each press can produce only the parts assigned to it (see figure 6(a)). In order for one press to produce the parts of the other's additional tooling is need which is not available and it requires 
unacceptably long time to be available (more than two months) and relatively high investment cost; thus such an option is rejected.

- Press - CNC: The department consists of one press and one CNC. In this case, the CNC machine may produce the parts normally produced by the press by reloading the proper NC program (see figure 6(b)).

- $\mathrm{CNC}-\mathrm{CNC}$ : In this case the, parts produced in one machine can be produced by the other machine by loading the proper NC program (see figure 6(c)).

The assignment of the parts to the machines is shown in the figures below. The punching department processes six different parts of the refrigerator.

[Insert figure 6 about here]

The processing time required by each machine to process a part is given in table 3 .

[Insert table 3 about here]

The breakdown profile for each of machine type is given in table 4 and the capacity of each of the three configurations is given in table 5. The department operates in two shifts of 7.5 hours each.

[Insert tables 4 and 5 about here]

The input per day for each system is given by a normal distribution with mean value the capacity of the system and standard deviation Std.Dev. $=20$. The experiments 
have been executed for 10 different input profiles, for all three systems for a period of 90 days with the sampling period to be per day. The results are shown in table 6 and they present that the third solution has the highest flexibility. Its responsiveness is expressed by its ability to keep FTs in low levels, although the PTs of the parts are considerably higher. The results present that FLEXIMAC can be used for measuring the flexibility of the punching department.

[Insert table 6 about here]

\section{Extended enterprise}

The FLEXIMAC measure, in the previous paragraphs, has been applied to a 'traditional' shop-floor environment. Its application under more complex production environments, such as the extended enterprise, is also examined hereafter. The extended enterprise expands beyond traditional organizational boundaries. It includes the relationships that an enterprise has with its customers, suppliers and business partners (Jagdev and Browne 1998, Chryssolouris et. al. 2004). In enterprise manufacturing level, and more specifically, for multi-product manufacturers (as in the automotive industry), the decisions of allocating products to plants are of major importance, since they bound the manufacturer's ability to meet target demand and achieve objectives (Chandra et al. 2005). The ability of plants to produce concurrently more than one product (product mix flexibility) provides the manufacturer with some degree of flexibility regarding the assignment of products to plants. In such cases, FLEXIMAC could be used for evaluating the flexibility of alternative plant-product assignment configurations. Performance evaluation in the extended enterprise by using FLEXIMAC, could take 
place either during the design phase of a production network or during the operational phase in order for the flexibility of alternative production networks operating in the extended enterprise to be compared. However, in the production network level, the calculation of processing as well as the flow (or lead) time is more complex, due to the involvement of a number of different stakeholders (OEMs, suppliers, distribution centres, dealers etc). The demand excitation triggers different entities in the enterprise that have to respond and coordinate their actions. Consequently, for the calculation of the processing time, the immediate steps, either parallel or serial, allocated to the different nodes of the production network, should be considered. The lead-times practically achieved are usually higher than the 'designed' ones, mainly due to the supply chain deficiencies, such as delays in the supply of materials or subassemblies, synchronization in the available capacities, capacity planning delays, reliability of exchanged information, delays occurring internally in the individual nodes of the production network etc.

\section{Conclusions}

The results of the simulation experiments indicate that there is a strong correlation between the values of FLEXIMAC and the actual flexibility of a manufacturing system, both in simple 'laboratory' systems and in real industrial cases. The variations in the configuration of a system have a measurable effect on the value of the indicator. If we increase the flexibility of the system then the value of the indicator will also be increased reflecting the changes in the system's flexibility. FLEXIMAC can be used in the design phase for evaluating alternative manufacturing systems solutions. This is achieved by exciting the designs, in simulation mode, to different input demands and 
recording their response, in the same manner as it is presented in the case studies of this work. Apart from the design phase, FLEXIMAC could also be used in the operating phase of production systems for comparing for example, the flexibility performance of similar departments, in the different production sites of a company, by utilizing orders data and production data usually available in the modern ERP systems. Thus, FLEXIMAC could be integrated into an industrial IT system and could provide a 'live indication' of manufacturing flexibility, based on input/output material and information data flow. As an example, an automotive industry could use this measure to compare the flexibility of different assembly lines located in different production sites.

The input and output datasets for the calculation of the indicator do not depend on the type of flexibility being examined. Therefore, a further topic of investigation will be the study of this indicator's relationship to the different types of flexibility, such as those of product and volume. Moreover, FLEXIMAC will be used in the context of analyzing and evaluating different control and scheduling policies, since they have a direct impact on the system's flexibility.

\section{Acknowledgements}

This study was partially supported by the IMS RTD project LICOPRO/IST-2001-37603 and the RTD project X-CHANGE, both funded by the European Commission.

\section{References}

Alexopoulos, K., Mamassioulas, A., Mourtzis, D. and Chryssolouris, G., Volume and product flexibility: a case study for a refrigerators producing facility, 10th IEEE 
International Conference on Emerging Technologies and Factory Automation, Catania, Italy, 19-22 September 2005, 891-897.

Barad, M. and Nof, S.Y, CIM flexibility measures: A review and a framework for analysis and applicability assessment. Int. j. Computer Integrated Manufacturing, 1997, 10, 296- 308

Bateman, N., Stockton, D. J. and Lawrence, P., Measuring the mix response flexibility of manufacturing systems. International Journal of Production Research, 1999, 37, $871-880$

Beach, R., Muhlemann, A. P., Price, D. H. R., Paterson, A. and Sharp, J. A., A review of manufacturing flexibility. European Journal of Operational Research, 2000, 122, $41-57$.

Bengtsson, J. and Olhager, J., Valuation of product-mix flexibility using real options. Int. J. Production Economics, 2002, 78, 13-28.

Beskese, A., Kahraman, C. and Irani, Z., Quantification of flexibility in advanced manufacturing systems using fuzzy concept, International Journal of Production Economics, 2004, 89, 45-56.

Borenstein, D., A directed acyclic graph representation of routing manufacturing flexibility. European Journal of Operational Research, 127, 2000, 78-93.

Boyer, K.K. and Leong, K.G., Manufacturing flexibility at the plant level. Omega, 1996, 24, 495-510.

Brill, P. H. and Mandelbaum, M., On measures of flexibility in manufacturing systems. International Journal of Production Research, 1989, 27, 747-756.

Browne, J., Dubois, D., Rathmill, K., Sethi, S. P. and Stecke, K. E., Classification of Flexible Manufacturing Systems. The FMS Magazine, 1984, 2, 114-117. 
Chandra, C., Everson, M. and Grabis, J., Evaluation of enterprise-level benefits of manufacturing flexibility. Omega, 2005, 33, 17 - 31 .

Chang, A.Y, Whitehouse, D.J., Chang S.L. and Hsieh, Y.C., An approach to the measurement of single-machine Flexibility, Int. J. Prod. Res., 2001, 39, 1589-1601.

Chryssolouris, G., Manufacturing Systems - Theory and Practice, $2^{\text {nd }}$ Edition, 2005, (Springer-Verlag: New York).

Chryssolouris, G. and Lee, M., An assessment of flexibility in manufacturing systems. Manufacturing Review, 1992, 5, 105-116.

Chryssolouris, G., Flexibility and its measurement. Proceedings of the CIRP Annals, 1996,45, 581-587.

Chryssolouris, G., Anifantis, N. and Karagiannis, S., An approach to the dynamic modelling of manufacturing systems. International Journal of Production Research, 1998, 36, 475-483.

Chryssolouris, G., Makris, S., Xanthakis, V. and Mourtzis, D., Towards the internet based supply chain management for the shiprepair industry. International Journal of Computer Integrated Manufacturing, 2004, 17, 45-57.

De Toni, A. and Tonchia, S., Manufacturing flexibility: a literature review, International Journal of Production Research, 1998, 36, 1587-1617.

Garavelli A.C., Performance analysis of a batch production system with limited flexibility. International Journal of Production Economics, 2001, 69, 39- 48.

Gupta, D., On measurement and valuation of manufacturing flexibility. International Journal of Production Research, 1993, 31, 2947-2958.

Hop, N.V., Approach to measure the mix response flexibility of manufacturing systems. Int. J. Prod. Res., 2004, 42, 1407-1418. 
Jagdev, H. S. and Browne, J., The extended enterprise - a context for manufacturing. Production Planning \& Control, 1998, 9, 216- 229.

Khouja, M., An aggregate production planning framework for the evaluation of volume flexibility. Production Planning \& Control, 1998, 9, 127- 137.

Koren, Y., Maier-Speredelozzi, V. and Hu, S.J., Convertibility measures for manufacturing systems, Annals of the CIRP, 2003, 52, 367-370.

Kumar, V., Entropic measures of manufacturing flexibility. International Journal of Production Research, 1987, 25, 957-966.

Kurtoglu, A., Flexibility analysis of two assembly lines, Robotics and ComputerIntegrated Manufacturing, 2004, 20, 247-253.

Ramasesh, R., Kulkarni, S. and Jayakumar, M., Agility in manufacturing systems: An exploratory modeling framework and simulation, Integrated Manufacturing Systems, 2001, 12, 534-548.

Seybert, A. F., Estimation of damping from response spectra. Journal of Sound and Vibration, 1981, 75, 199-206.

Shewchuk, J., A set of generic flexibility measures for manufacturing applications. International Journal of Production Research, 1999, 37, 3017-3042.

Stecke, K.E. and Browne, J., Variations in Flexible Manufacturing Systems According to the Relevant Types of Automated Materials Handling. Material Flow, 1985, 2, $179-185$.

Tsourveloudis, N. and Phillis, Y., Fuzzy assessment of machine flexibility. IEEE Transactions on Engineering Management, 1998, 45, 78-87.

Wahab, M. I. M., Measuring machine and product mix flexibilities of a manufacturing system. International Journal of Production Research, 43, 2005, 3773-3786. 
Wiendahl, H.P, Heger, C.L., Justifying changeability. A methodological approach to achieving cost effectiveness. The manufacturing Journal for Manufacturing Science and Production, 2004, 6, 33-39. 


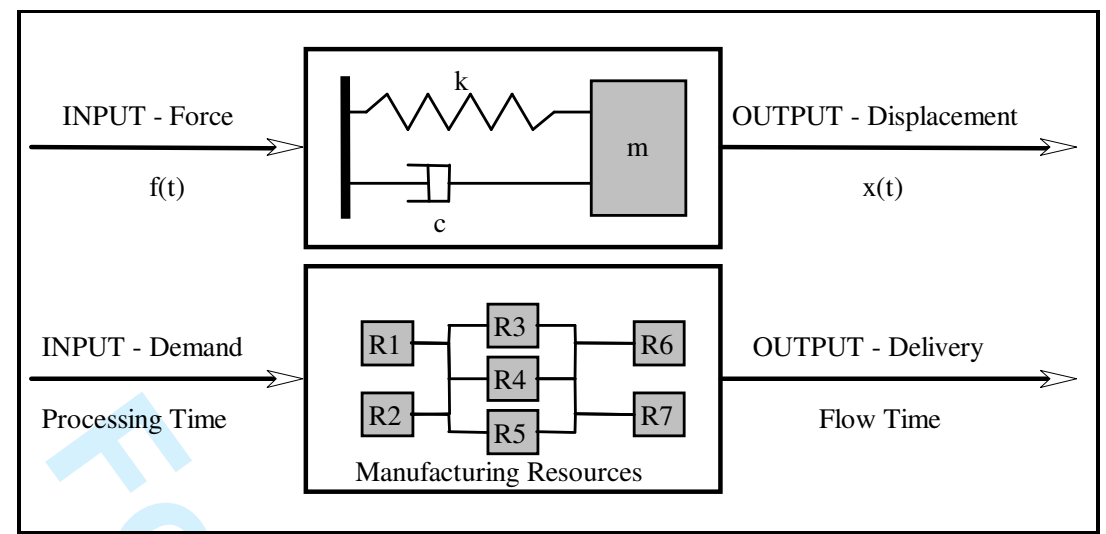

Figure 1: Input/output in a mechanical and a manufacturing system. 


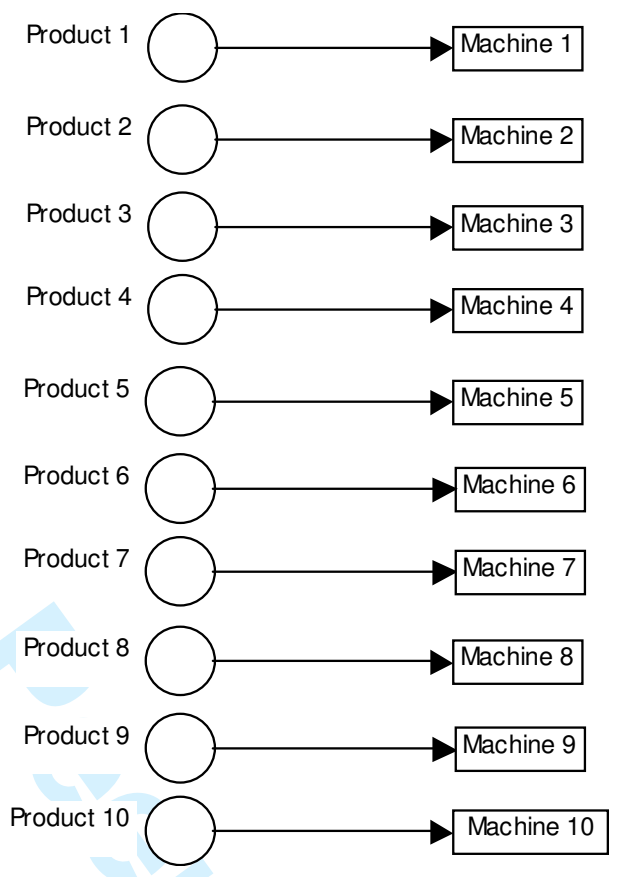

Figure 2a: Configuration A with lowest flexibility: each machine can process only one product. 


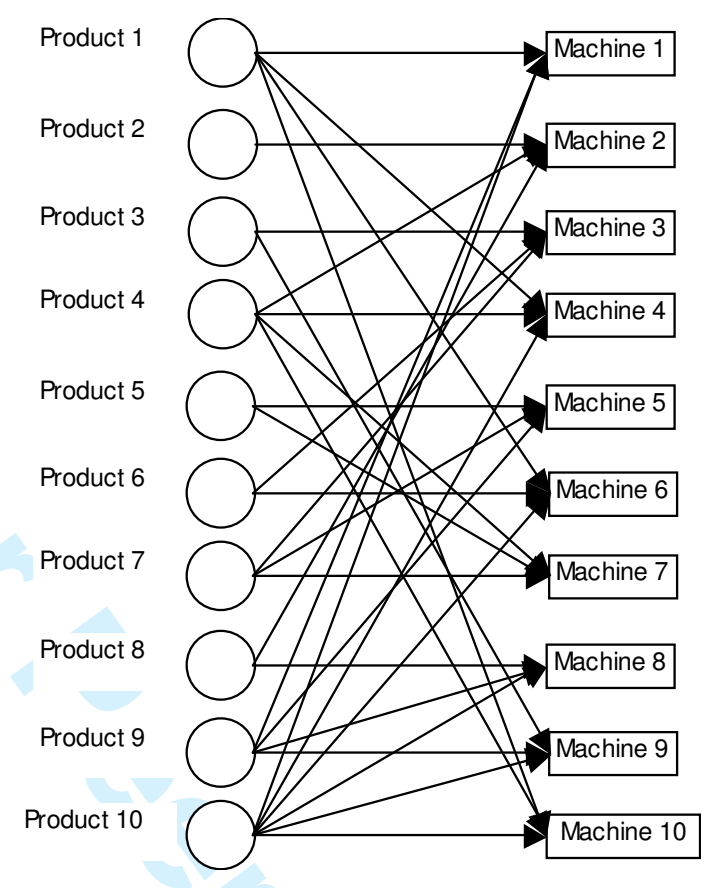

Figure 2b: Configuration B with medium flexibility: each machine can process three products. 


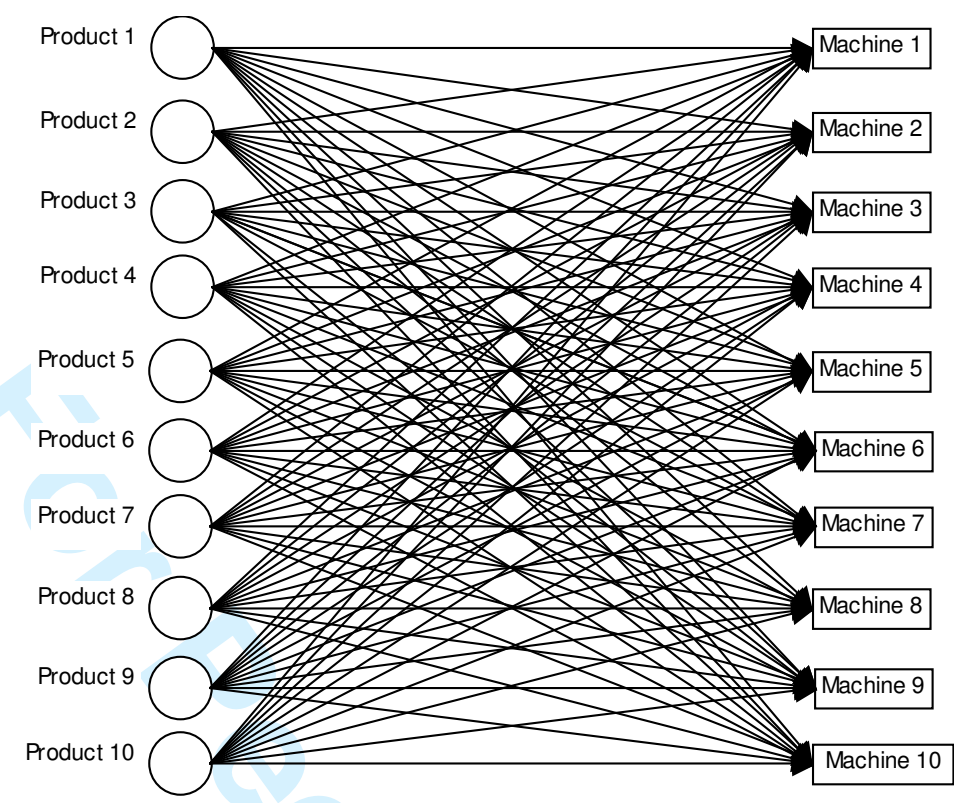

Figure 2c: Configuration $\mathrm{C}$ with maximum flexibility: each machine can process ten products. 


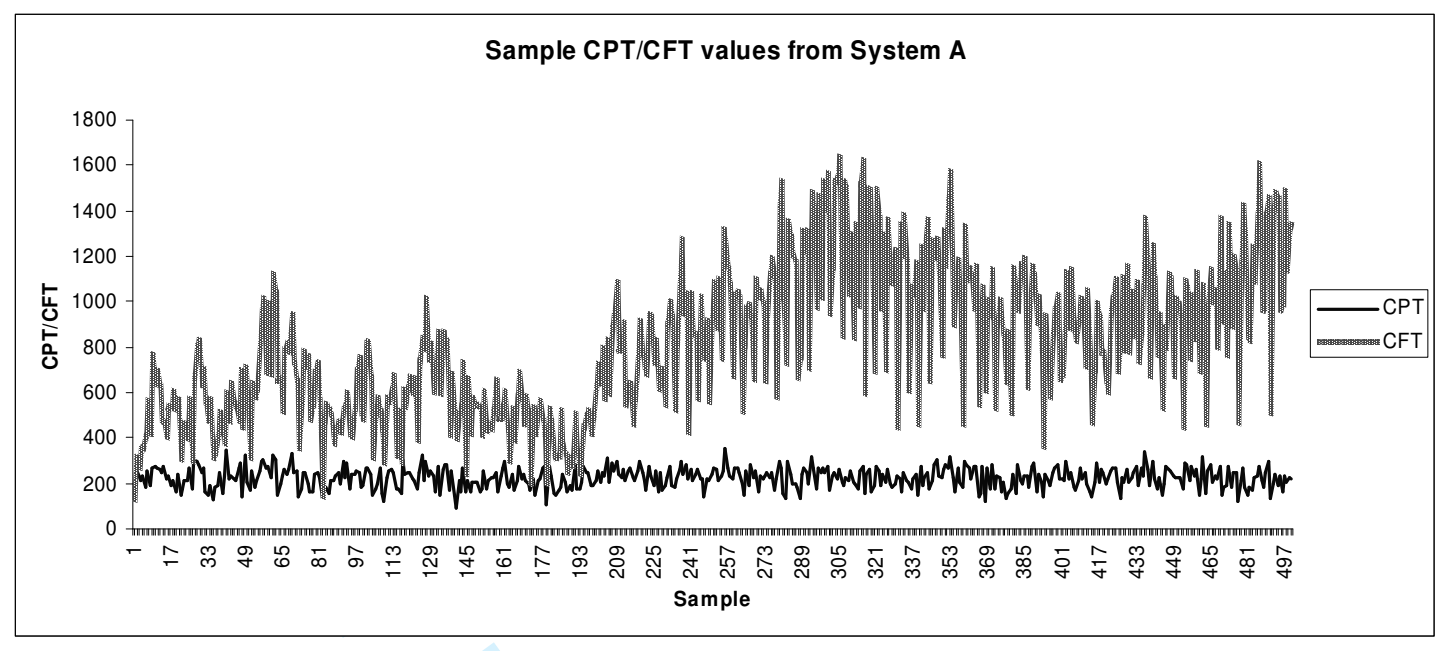

Figure 3: An example plot of 500 CPT/CFT samples. 


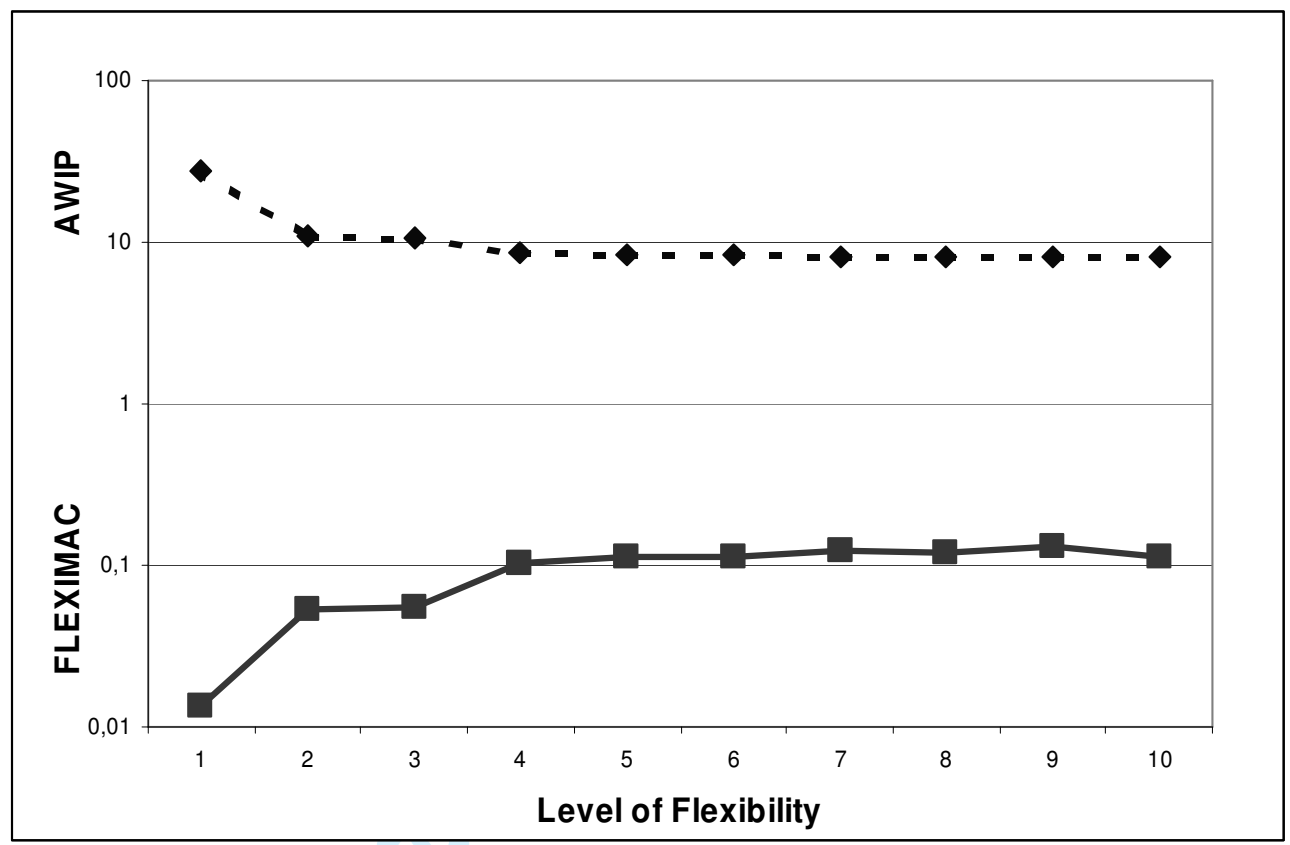

Figure 4: FLEXIMAC and AWIP values for different levels of flexibility. 


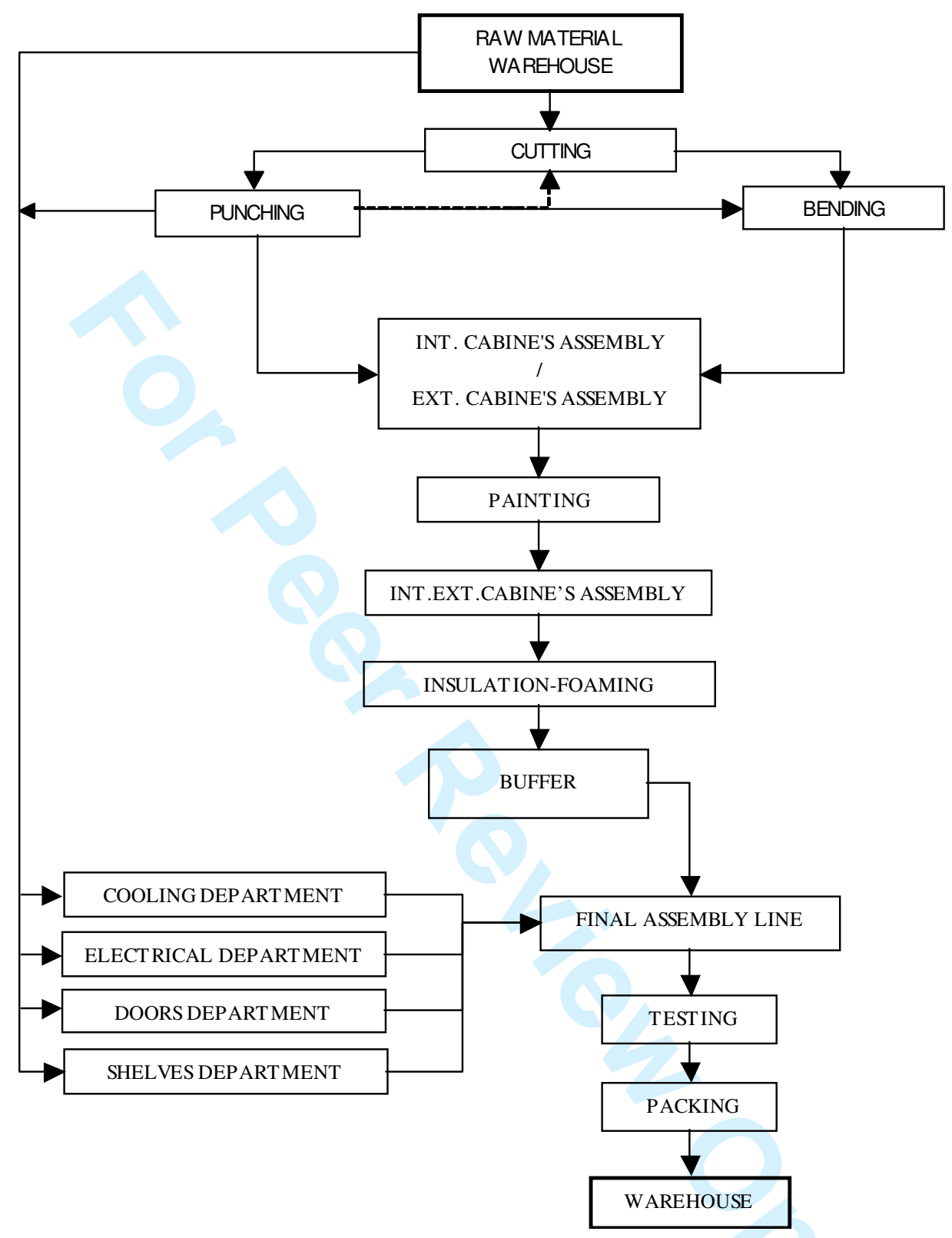

Figure 5: Schematic diagram of the real manufacturing system. 


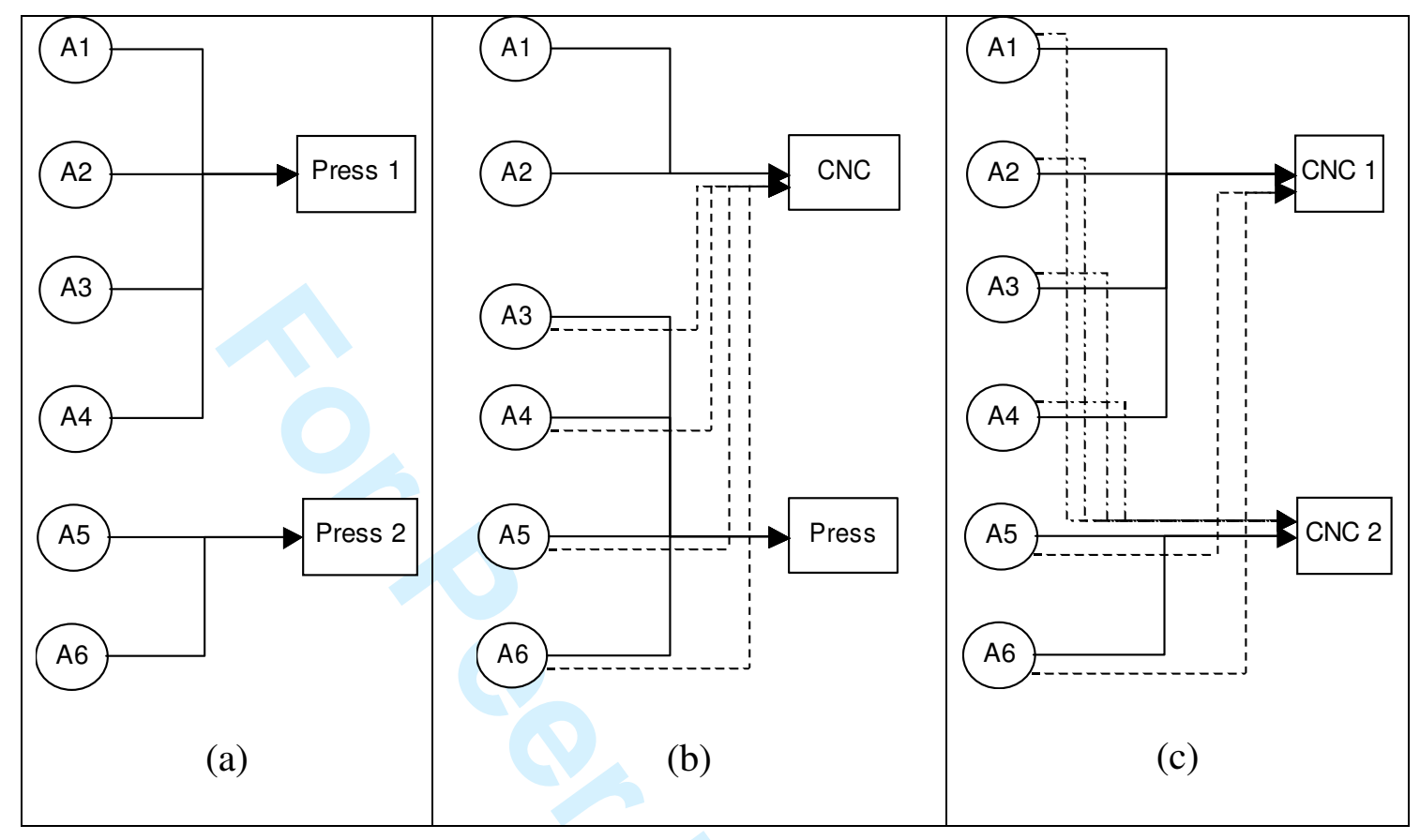

Figure 6: Possible solutions for the punching department. 


\begin{tabular}{cccc}
\hline \multicolumn{4}{c}{ Simulation Results } \\
\hline Simulation Run & $\begin{array}{c}\text { Low } \\
\text { Flexibility }\end{array}$ & $\begin{array}{c}\text { Medium } \\
\text { Flexibility }\end{array}$ & $\begin{array}{c}\text { High } \\
\text { Flexibility }\end{array}$ \\
\hline 1 & 0.0122 & 0.0442 & 0.1356 \\
2 & 0.0189 & 0.0542 & 0.1043 \\
3 & 0.0065 & 0.0553 & 0.1249 \\
4 & 0.0113 & 0.0646 & 0.1475 \\
5 & 0.0168 & 0.0698 & 0.0939 \\
6 & 0.0089 & 0.0430 & 0.1083 \\
7 & 0.0188 & 0.0527 & 0.0965 \\
8 & 0.0103 & 0.0433 & 0.1251 \\
9 & 0.0156 & 0.0508 & 0.0991 \\
10 & 0.0151 & 0.0802 & 0.0827 \\
\hline Mean & $\mathbf{0 . 0 1 3 4}$ & $\mathbf{0 . 0 5 5 8}$ & $\mathbf{0 . 1 1 1 8}$ \\
Std. Deviation & 0.0040 & 0.0117 & 0.0195 \\
\hline
\end{tabular}

Table 1: FLEXIMAC values, mean value and standard deviation for each configuration and simulation run. 


\begin{tabular}{cccc}
\hline \multicolumn{4}{c}{ Simulation Results } \\
\hline Simulation Run & $\begin{array}{c}\text { Low } \\
\text { Flexibility }\end{array}$ & $\begin{array}{c}\text { Medium } \\
\text { Flexibility }\end{array}$ & High Flexibility \\
\hline 1 & 0.0026 & 0.0072 & 0.0092 \\
2 & 0.0019 & 0.0109 & 0.0133 \\
3 & 0.0053 & 0.0072 & 0.0103 \\
4 & 0.0060 & 0.0089 & 0.0116 \\
5 & 0.0034 & 0.0090 & 0.0097 \\
6 & 0.0022 & 0.0055 & 0.0156 \\
7 & 0.0024 & 0.0039 & 0.0112 \\
8 & 0.0051 & 0.0098 & 0.0173 \\
9 & 0.0041 & 0.0082 & 0.0160 \\
10 & 0.0013 & 0.0138 & 0.0146 \\
\hline Mean & $\mathbf{0 . 0 0 3 4}$ & $\mathbf{0 . 0 0 8 4}$ & $\mathbf{0 . 0 1 2 9}$ \\
Std. Deviation & 0.0015 & 0.0026 & 0.0027 \\
\hline
\end{tabular}

Table 2: FLEXIMAC mean value and standard deviation for each configuration when breakdowns are considered. 


\begin{tabular}{cccc}
\hline Part & Part Description & $\begin{array}{l}\text { Press } \\
(\mathrm{min})\end{array}$ & $\begin{array}{c}\text { CNC } \\
(\mathrm{min})\end{array}$ \\
\hline A1 & Cabin's base & 0.33 & 0.75 \\
A2 & Cabin's bottom & 0.30 & 0.72 \\
A3 & Inner liner bottom & 0.33 & 0.67 \\
A4 & Canopy & 0.27 & 0.68 \\
A5 & Inner liner side & 0.67 & 2.33 \\
A6 & Inner liner top & 0.33 & 0.67 \\
\hline Total & & 2.23 & 5.82 \\
\hline
\end{tabular}

Table 3: Processing time of parts on each type of machine. 


\begin{tabular}{ccc}
\hline & $\begin{array}{c}\text { MTBF } \\
(\min )\end{array}$ & $\begin{array}{c}\text { MTTR } \\
(\min )\end{array}$ \\
\hline Press & 19150 & 240 \\
CNC & 57600 & 20 \\
\hline
\end{tabular}

Table 4: Machine breakdowns profile. 


\begin{tabular}{cccc}
\hline & Press-Press & CNC-Press & CNC-CNC \\
\hline $\begin{array}{c}\text { Capacity } \\
\text { (Parts/Day) }\end{array}$ & 730 & 570 & 300 \\
\hline
\end{tabular}

Table 5: Capacity of each configuration. 


\begin{tabular}{cccc}
\hline \multicolumn{4}{c}{ Real Case Study Results } \\
\hline Simulation Run & Press-Press & Press-CNC & CNC-CNC \\
\hline 1 & $1.48 \mathrm{E}-05$ & $2.55 \mathrm{E}-05$ & $13.9 \mathrm{E}-05$ \\
2 & $2.09 \mathrm{E}-05$ & $2.99 \mathrm{E}-05$ & $16.8 \mathrm{E}-05$ \\
3 & $1.10 \mathrm{E}-05$ & $1.84 \mathrm{E}-05$ & $14.2 \mathrm{E}-05$ \\
4 & $1.80 \mathrm{E}-05$ & $5.34 \mathrm{E}-05$ & $17.4 \mathrm{E}-05$ \\
5 & $1.59 \mathrm{E}-05$ & $6.79 \mathrm{E}-05$ & $7.23 \mathrm{E}-05$ \\
6 & $2.71 \mathrm{E}-05$ & $4.24 \mathrm{E}-05$ & $13.4 \mathrm{E}-05$ \\
7 & $1.11 \mathrm{E}-05$ & $2.73 \mathrm{E}-05$ & $7.41 \mathrm{E}-05$ \\
8 & $1.66 \mathrm{E}-05$ & $2.56 \mathrm{E}-05$ & $6.11 \mathrm{E}-05$ \\
9 & $1.30 \mathrm{E}-05$ & $8.38 \mathrm{E}-05$ & $11.9 \mathrm{E}-05$ \\
10 & $1.29 \mathrm{E}-05$ & $4.11 \mathrm{E}-05$ & $10.3 \mathrm{E}-05$ \\
\hline Mean & $\mathbf{1 . 6 1 E}-05$ & $\mathbf{4 . 2 E}-05$ & $\mathbf{1 2 . 0 E}-05$ \\
Standard & $0.49 \mathrm{E}-05$ & $2.1 \mathrm{E}-05$ & $4.0 \mathrm{E}-05$ \\
Deviation & &
\end{tabular}

Table 6: Real industrial system case study results. 


\section{List of Figures}

Figure 1: Input/output in a mechanical and a manufacturing system.

Figure 2a: Configuration A with lowest flexibility: each machine can process only one product.

Figure 2b: Configuration B with medium flexibility: each machine can process three products.

Figure 2c: Configuration $\mathrm{C}$ with maximum flexibility: each machine can process ten products.

Figure 3: An example plot of 500 CPT/CFT samples.

Figure 4: FLEXIMAC and AWIP values for different levels of flexibility.

Figure 5: Schematic diagram of the real manufacturing system.

Figure 6: Possible solutions for the punching department. 